\title{
DOE Office of Indian Energy: Leveraging Tribal Resources to Support Department of Defense Strategic Energy Goals
}

The U.S. Department of Defense (DoD) has announced several strategic energy goals including obtaining $25 \%$ of its energy from renewable energy and promoting energy security. Indian Tribes are uniquely situated to help the DoD achieve these goals. According to DoD, there are more than 50 Tribes located adjacent to or near military installations.

The DOE Office of Indian Energy has begun exploring how to leverage the considerable renewable energy resources in Indian Country to help the DoD achieve some of its strategic energy goals, including educating tribal leaders and military leaders on energy development partnerships on Indian lands, analyzing and mapping the most promising partnership opportunities, and working with the National Renewable Energy Laboratory (NREL) to provide technical assistance to Tribes on working with local military installations. NREL estimates that Indian lands have the following amounts of renewable energy generation potential:

\begin{tabular}{|l|l|}
\hline Tribal Technology Generation Potential & Megawatt Hours (MWh) \\
\hline Wind & $26,059,675$ \\
\hline Solar: Photovoltaic (PV) (Utility-Scale, Rural) & $4,372,566,050$ \\
\hline Solar: PV (Utility-Scale, Urban) & $8,432,273$ \\
\hline Solar: Concentrating Solar Power & $4,293,336,157$ \\
\hline Geothermal: Enhanced Geothermal System & $2,007,195,000$ \\
\hline Geothermal: Hydrothermal & $1,805,368$ \\
\hline Biomass: Solid & 675,136 \\
\hline Biomass: Gaseous & 592,238 \\
\hline Hydropower & $2,605,585$ \\
\hline $\begin{array}{l}{ }^{1} \text { Based on methodology from Lopez, A. et al. (2012). Forthcoming report to be published } \\
\text { by the National Renewable Energy Laboratory. }\end{array}$ \\
\hline Analysis assumes development of single technology across all available land.
\end{tabular}

Of the Tribes identified as being located in close proximity to military bases, $54 \%$ are located in the West, $18 \%$ in the Pacific Northwest, and 12\% in New England and New York.
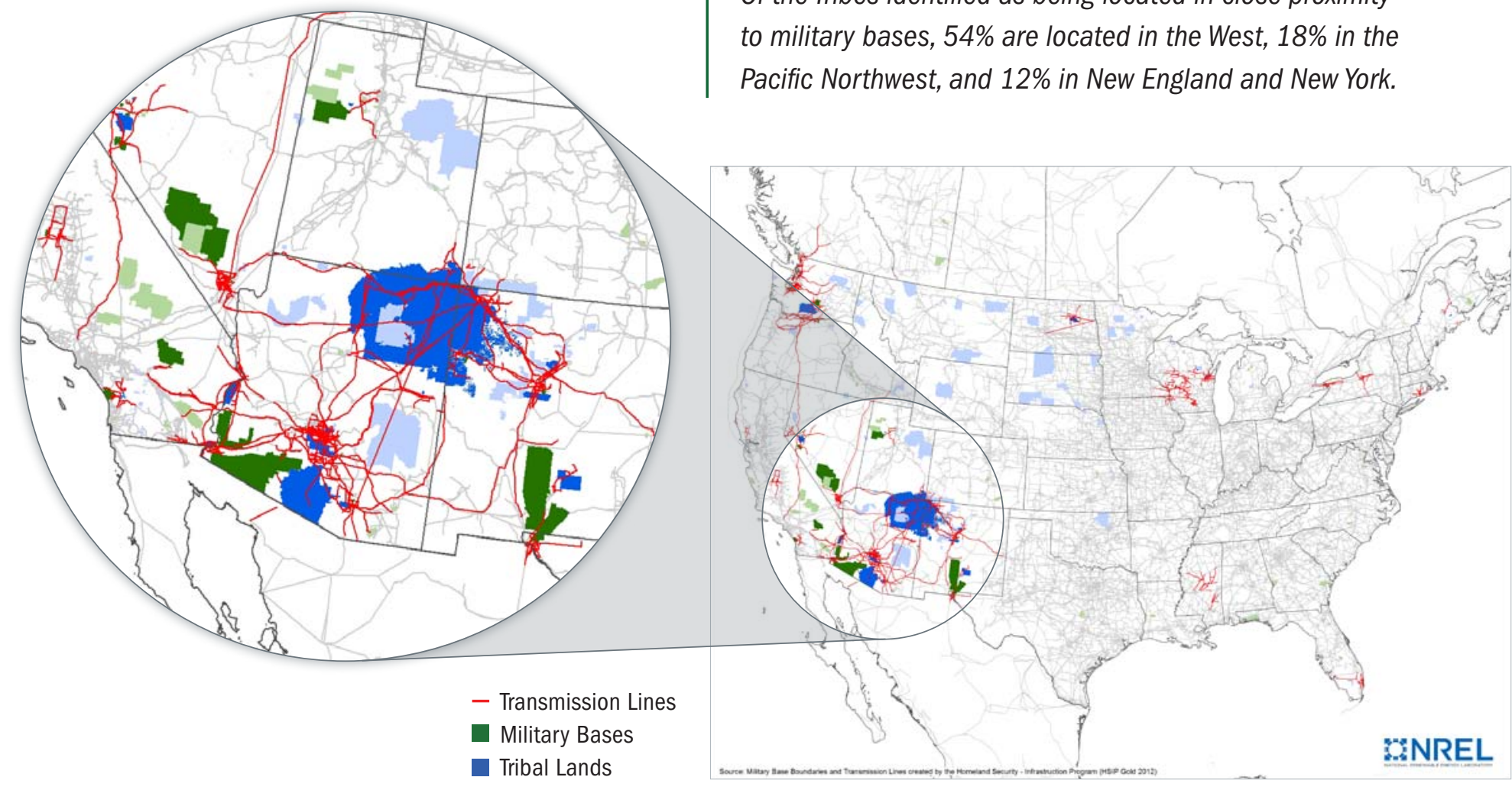

MNREL 


\section{Tribes Located Near Military Bases}

There are 53 reservations located within 10 miles of 65 military installations. There are 20 reservations located near multiple bases. For example, the Mashantucket Pequot reservation is within 10 miles of the Coast Guard Academy and a Naval Submarine Base.

\begin{tabular}{|c|c|c|c|}
\hline Tribe & Military Bases & Tribe & Military Bases \\
\hline \multicolumn{2}{|l|}{ Arizona } & \multicolumn{2}{|l|}{ Maine } \\
\hline \multirow[t]{2}{*}{ Cocopah } & \multirow{2}{*}{$\begin{array}{l}\text { Barry M Goldwater } \\
\text { Air Force Range } \\
\text { USMC Air Station-Yuma }\end{array}$} & $\begin{array}{l}\text { Passamaquoddy Tribe } \\
\text { of Indian Township* ** }\end{array}$ & Columbia Air Force Station \\
\hline & & \multicolumn{2}{|l|}{ Mississippi } \\
\hline \multirow[t]{3}{*}{ Colorado River } & Yuma Proving Ground & \multirow{2}{*}{ Mississippi Choctaw } & \multirow{2}{*}{$\begin{array}{l}\text { Navy Outlying Field } \\
\text { Joe Williams }\end{array}$} \\
\hline & Barry M Goldwater & & \\
\hline & Air Force Range & \multicolumn{2}{|l|}{ Nevada } \\
\hline \multirow[t]{2}{*}{ Fort Yuma (Quechan) } & $\begin{array}{l}\text { National Guard Rifle Range } \\
\text { USMC Air Station-Yuma }\end{array}$ & Carson Colony & $\begin{array}{l}\text { Military Department } \\
\text { Complex ANG }\end{array}$ \\
\hline & Yuma Proving Ground & Fallon & Naval Air Station Fallon \\
\hline Gila Bend & $\begin{array}{l}\text { Barry M Goldwater } \\
\text { Air Force Range }\end{array}$ & \multirow{2}{*}{ Fallon Colony } & $\begin{array}{l}\text { Fallon Naval Air Station } \\
\text { Bombing Range }\end{array}$ \\
\hline \multirow[b]{2}{*}{ Gila River } & $\begin{array}{l}\text { Casa Grande Military } \\
\text { Reservation }\end{array}$ & & Naval Air Station Fallon \\
\hline & Florence Military Reservation & \multirow{3}{*}{ Las Vegas Colony } & $\begin{array}{l}\text { Clark County Army } \\
\text { National Guard }\end{array}$ \\
\hline Pascua Yaqui** & Davis-Monthan Air Force Base & & $\begin{array}{l}\text { Nellis Air Force Base } \\
\text { Nellis Air Force Range }\end{array}$ \\
\hline \multirow{2}{*}{ Salt River } & \multirow{2}{*}{$\begin{array}{l}\text { Papago Park Military } \\
\text { Reservation }\end{array}$} & & Nellis Small Arms Range \\
\hline & & \multirow{3}{*}{ Walker River* } & Fallon Naval Air Station \\
\hline San Xavier & $\begin{array}{l}\text { Davis-Monthan Air Force Base } \\
\text { Sahuarita Air Force Range }\end{array}$ & & $\begin{array}{l}\text { Bombing Range } \\
\text { Hawthorne Army Depot }\end{array}$ \\
\hline \multirow{3}{*}{ Tohono O'odham* } & $\begin{array}{l}\text { Barry M Goldwater } \\
\text { Air Force Range }\end{array}$ & & $\begin{array}{l}\text { U.S. Naval Reservation- } \\
\text { Target Area }\end{array}$ \\
\hline & $\begin{array}{l}\text { Casa Grande Military } \\
\text { Reservation }\end{array}$ & Washoe & $\begin{array}{l}\text { Military Department } \\
\text { Complex ANG }\end{array}$ \\
\hline & Florence Military Reservation & \multicolumn{2}{|l|}{ New Mexico } \\
\hline \multicolumn{2}{|l|}{ British Columbia } & \multirow{2}{*}{ Cochiti Pueblo } & \multirow{2}{*}{$\begin{array}{l}\text { New Mexico National } \\
\text { Guard Armory }\end{array}$} \\
\hline Nooksack & CFB Esquimalt-Matsqui & & \\
\hline \multicolumn{2}{|l|}{ California } & Isleta Pueblo & Kirtland Air Force Base \\
\hline \multirow{2}{*}{$\begin{array}{l}\text { Manchester (Point } \\
\text { Arena) Rancheria }\end{array}$} & \multirow{3}{*}{ Point Arena Airforce Station } & Mescalero Apache & White Sands Missile Range \\
\hline & & Navajo* & Fort Wingate \\
\hline Middletown Rancheria & & & \\
\hline Pala & Camp Pendleton & Sandia Pueblo & Kirtland Air Force Base \\
\hline Pala & Marine Corps Base & Santo Dominøo Pueblo & New Mexico National \\
\hline Pechanga & Camp Pendleton & & Guard Armory \\
\hline Pechanga & Marine Corps Base & Ysleta Del Sur Pueblo & Fort Bliss \\
\hline Santa Rosa Rancheria & Lemoore Naval Air Station & Zuni Pueblo** & Fort Wingate Military \\
\hline Twenty-Nine Palms & Marine Corps AGCC & & Reservation \\
\hline Twerty-ovine ramis & Twentynine Palms & New York & \\
\hline Connecticut & & & Stockbridge Research Facility \\
\hline & New London Naval & Oneida (East) & Verona Research Facility \\
\hline Mashantucket Pequot & $\begin{array}{l}\text { Submarine Base } \\
\text { United States Coast Guard } \\
\text { Academy S }\end{array}$ & Onondaga & $\begin{array}{l}\text { Hancock Field Ang Base } \\
\text { USMC Reserve Training Center }\end{array}$ \\
\hline Florida & & Tuscarora & $\begin{array}{l}\text { Niagara Falls Air } \\
\text { Reserve Station }\end{array}$ \\
\hline & Navsea Warfare Center & North Dakota & \\
\hline Hollywood & $\begin{array}{l}\text { Fort Lauderdale } \\
\text { U.S. Coast Guard Station }\end{array}$ & Devils Lake Sioux & Camp Grafton \\
\hline & Fort Lauderdale & Ontario & \\
\hline Seminole Homestead & $\begin{array}{l}\text { Navsea Warfare Center } \\
\text { Fort Lauderdale }\end{array}$ & Tuscarora & $\begin{array}{l}\text { Department of } \\
\text { National Defense }\end{array}$ \\
\hline Trust Lands & U.S. Coast Guard Station & Rhode Island & \\
\hline & Fort Lauderdale & Narragansett & Fort Nathaniel Greene \\
\hline
\end{tabular}

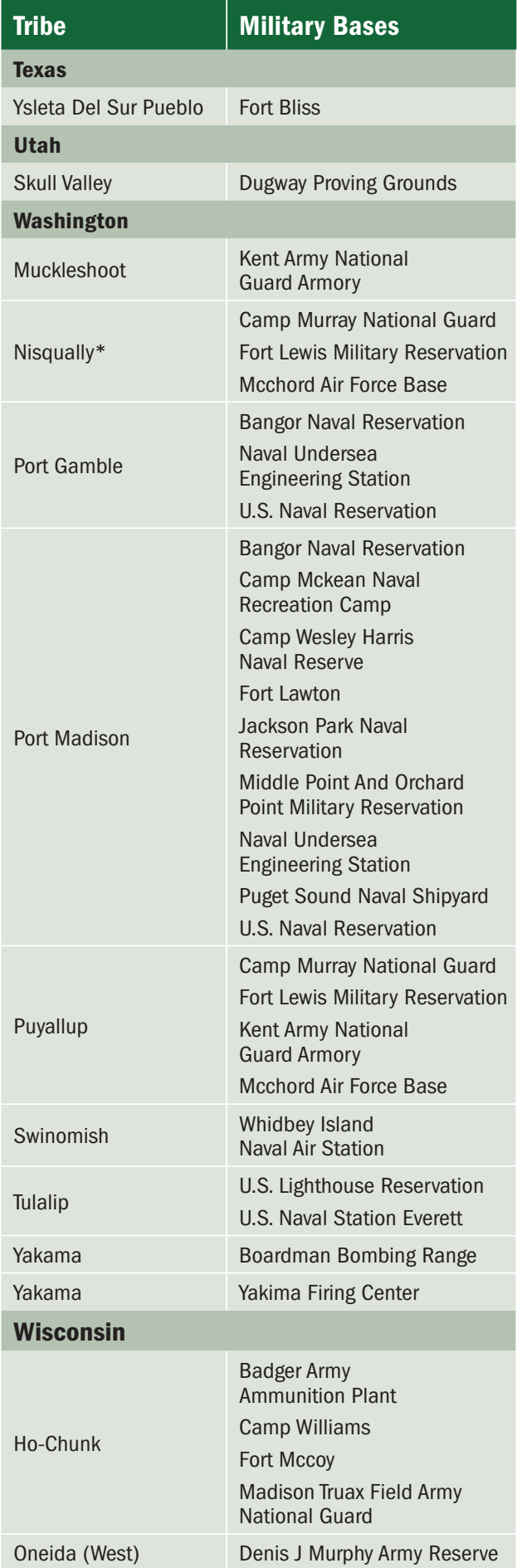

Oneida (West)

Kent Army National Guard Armory

Fort Lewis Military Reservation Mcchord Air Force Base

Naval Undersea Bangor Naval Reservation ckean Nava Camp Wesley Harris aval Reserve Jackson Park Nava Middle Point And Orchard Puget Sound Naval Shipyard Reservation Mcchord Air Force Base U.S. Lighthouse Reservation Everett ardman Bombing Range \begin{tabular}{ll|l} 
U.i. & Office of \\
Indian Energartment of
\end{tabular} energy.gov/indianenergy | indianenergy@hq.doe.gov November $2012 \cdot$ DOE/IE-0009

Printed with a renewable-source ink on paper containing at least $50 \%$ wastepaper, including $10 \%$ post consumer waste. 